\title{
25-Hydroxyvitamin D deficiency is a risk factor for atherosclerotic cardiovascular disease: a prospective study
}

\section{Shan Su}

Lanzhou Medical College: Lanzhou University

\section{Liting Wang}

Lanzhou University First Affiliated Hospital

\section{Xulei Tang}

Lanzhou University First Affiliated Hospital

\section{Nan Zhao}

Lanzhou University First Affiliated Hospital

\section{Conghui Guan}

Lanzhou University First Affiliated Hospital

\section{Hongli Li}

Lanzhou University First Affiliated Hospital

\section{Lijuan Liu}

Lanzhou University First Affiliated Hospital

JinJin Liu

Lanzhou University First Affiliated Hospital

\section{Hongxia Che}

Gansu Provincial Hospital

\section{Di Zhang}

Lanzhou Medical College: Lanzhou University

\section{Qiangmei Wang}

Lanzhou Medical College: Lanzhou University

\section{Songbo Fu}

Lanzhou University First Affiliated Hospital

Donghu Zhen ( $\nabla$ zhdh8279@163.com )

Lanzhou University First Affiliated Hospital

\section{Original investigation}

Keywords: Type 2 diabetes mellitus, Atherosclerotic cardiovascular disease, 25-hydroxyvitamin D 
Posted Date: February 23rd, 2021

DOl: https://doi.org/10.21203/rs.3.rs-218364/v1

License: (c) (i) This work is licensed under a Creative Commons Attribution 4.0 International License. Read Full License 


\section{Abstract}

Background Vitamin D, a fat-soluble vitamin, play a protective role in the cardiovascular system. Increasing evidence has suggested that the vitamin D deficiency is a significant risk factor for atherosclerotic cardiovascular disease (ASCVD). The aim of the present study was to investigate the correlation between serum 25-hydroxyvitamin $D[25(\mathrm{OH}) \mathrm{D}]$ and the risk of ASCVD in Chinese, especially in people with type 2 diabetes mellitus(T2DM). Methods Based on the "REACTION" study conducted in 2011, 9014 residents aged 40 to 75 in Lanzhou, Gansu province were followed up from 2014 to 2016. A total of 7061 subjects with complete data from the two surveys in present study were analyzed. Baseline population was classified into four groups based on 25(OH)D quartiles, comparing the different $25(\mathrm{OH}) \mathrm{D}$ groups of ASCVD incidence after follow-up, multivariate Cox proportional hazards regression modelling was used to estimate associations between 25(OH)D levels and the risk of ASCVD and to further analyze the relationship between ASCVD risk and 25(OH)D levels in different glucose metabolism status. Results Among 7,061 subjects aged 40 to 75 years, the median serum 25(OH)D level was $15.91 \mathrm{ng} / \mathrm{ml}$, and the prevalence of vitamin D deficiency was $75.1 \%$, merely $2.0 \%$ of those with vitamin D sufficiency. During the follow-up period of 3.3 years, 216 cases of ASCVD events were recorded. The cumulative incidence of ASCVD from lowest of 25(OH)D levels to the highest were $4.1 \%, 3.0 \%, 3.1 \%$ and $2.0 \%$, respectively. Cox regression analysis indicated that the individuals in quartile 1 but not quartile 2 or 3 of 25(OH)D level had significantly higher rate of ASCVD than that in highest quartile, additionally, $10 \mathrm{ng} / \mathrm{ml}$ per increase in serum 25(OH)D at baseline, the ASCVD risk was reduced by 24\%[hazard ratio(HR):0.761, 95\% confident interval(Cl):0.590- 0.980, $\mathrm{P}<0.05]$. When the effects of 25(OH)D on ASCVD risk was assessed in different glucose metabolism status, the results showed that the low 25(OH)D plus T2DM was associated with high risk of ASCVD after adjustment of confounding risk factors (HR: 2.284, 95\% Cl: 1.242-4.202, P $<0.05)$. The risk of ASCVD decreased by $35 \%$ as serum $25(\mathrm{OH}) \mathrm{D}$ increased by $10 \mathrm{ng} / \mathrm{ml}$, which remained significant after stepwise adjustments $(P<0.05)$. Conclusions Serum 25(OH)D is independently and inversely associated with the risk of ASCVD in Chinese.Increasing vitamin $D$ levels can effectively reduce the risk of ASCVD incidence, especially in T2DM populations.

\section{Background}

Atherosclerotic cardiovascular disease (ASCVD) is one of the main causes of death and morbidity globally in modern times, which is seriously endangers public health and brings heavy economic and living burden to society and family[1]. Diabetes mellitus, obesity, hyperlipidemia, hypertension, smoking and drinking are all risk factors for ASCVD[2]. In particular, type 2 diabetes mellitus(T2DM) is one of the most prominent risk factors for ASCVD and is considered as an equal risk disease for ASCVD[3].

Vitamin D, a fat-soluble vitamin, is well known for its pivotal role in calcium and phosphorus homeostasis and bone metabolism[4]. Vitamin D deficiency is a global health issue that affects more than one billion of children and adults worldwide and is now recognized as a pandemic[5]. Diet imbalance, insufficient sunlight exposure time, severe liver and kidney dysfunction, gastrointestinal absorption dysfunction, endocrine system disorder are the causes of vitamin D deficiency or insufficiency[6]. In addition to the 
classical regulation of calcium and phosphorus metabolism, recent studies have suggested that vitamin D may play a protective role in the cardiovascular system, it can not only regulate the immune system, inhibit the release of inflammatory factors, and protect vascular endothelial cells, but also inhibit coronary calcification, delay coronary atherosclerosis[7,8]. A growing body of literature has linked decreased serum vitamin D levels with cardiovascular diseases(CVD), particularly ASCVD $[9,10,11,12]$. However, large-scale prospective studies are lacking in China, the conclusions are inconsistent and the exact mechanisms of vitamin D and ASCVD are not clearly clarified. Thus, the available data highlights the need for further analysis of the associations between vitamin $D$ levels and ASCVD in Chinese.

In this study, participants aged 40 to 75 years from Lanzhou, Gansu province, who participated in the Risk Evaluation of Cancer in Diabetic Chinese Individuals $₫$ a Longitudinal『REACTIONखStudy in 2011 were enrolled. The key problems to be discussed in present study are as follows: firstly, to assessed the nutritional status of vitamin $D$ in middle-aged and elderly people in Lanzhou; secondly, to clarified the incidence of ASCVD with different levels of $25(\mathrm{OH}) \mathrm{D}$, and further to investigated the association between baseline serum 25(OH)D levels and the risk of ASCVD using a prospective cohort study method; finally, to analyzed the incidence of ASCVD at different glucose metabolism status and its correlation with serum $25(\mathrm{OH}) \mathrm{D}$ concentrations.

\section{Materials And Methods}

\section{Study population}

The Risk Evaluation of Cancers in Chinese Diabetic Individuals: A Longitudinal Study (REACTION Study) is a large, nationwide, prospective study, which conducted among 259,657 adults aged 40 years and older in 25 communities across mainland China from 2011 to 2012 in order to investigating the association of diabetes and cancer[13].The present work was a part of the REACTION study, which subjects aged 40-75 years were randomly selected from three communities in Lanzhou urban district, and nearly 9,014 individuals were enrolled in the original cohort. Participants with coronary heart disease, ischemic stroke or peripheral arterial disease at baseline were excluded from the analysis. After a median follow-up of 3.3 years, among 7061 eligible subjects were included in this study. The ASCVD events and deaths were confirmed by verifying the time and place of cardiovascular events or deaths, physical examination results and hospital certificates, etc. The study has been approved by the ethics committee of Shanghai Jiao Tong University. All study participants provided written informed consent (Figure 1).

\section{Clinical and laboratory measurements}

Data collection was conducted at community healthcare center by trained staff according to a standard protocol. All subjects were medically examined and interviewed using a standardized questionnaire to collect information on age, sex, residential region, visit date, family history, lifestyle(tea, coffee, alcohol, and smoking), dietary habits, physical activity level during leisure time, use of medications, self-reported diabetes, self-reported coronary heart disease, and self-reported stroke. 
Physical examination included measurement of height, weight and blood pressure. Body mass index (BMI) was calculated as weight in kilograms divided by height in metres squared. Blood pressure was consecutively measured in the non-dominant arm for three times.

Biochemical analyses such as fasting plasma glucose(FPG), $2 \mathrm{~h}$ plasma glucose (2h-PG), glycated haemoglobin ( $\mathrm{HbA1C}$ ), serum total cholesterol (TC), triglycerides(TG), high-density lipoprotein cholesterol(HDL-C) and low-density lipoprotein cholesterol(LDL-C) were performed at a laboratory in The First Hospital of Lanzhou University (Lanzhou, western China) with routine standardized methodologies on fresh samples of blood, which were obtained from all participants after overnight fasting (at least $8 \sim 10 \mathrm{~h}$ ) or $2 \mathrm{~h}$ in $75-\mathrm{g}$ oral glucose tolerance test (OGTT). Glucose was measured in plasma, and all other biochemical analyses were performed on serum. Serum 25(OH)D concentration was tested by enzymeimmunoassay (EIA; IDS Ltd, Boldon, UK). Clinical and laboratory definitions

According to the criteria established by the World Health Organization in 1999 [14], the subjects were categorized as follows: normal glucose tolerance[NGT:FPG $<6.1 \mathrm{mmol} / \mathrm{L}$ and $2 \mathrm{~h}-\mathrm{PG}<7.8 \mathrm{mmol} / \mathrm{L}$ ], impaired glucose regulation [IGR:6.1 mmol/L $\leq \mathrm{FPG}<7.0 \mathrm{mmol} / \mathrm{L}$ or $7.8 \mathrm{mmol} / \mathrm{L} \leq 2 \mathrm{hPG}<11.1$ $\mathrm{mmol} / \mathrm{L}$ ] and diabetes mellitus[DM:diagnosed diabetes and FPG $\geq 7.0 \mathrm{mmol} / \mathrm{L}$, or $2 \mathrm{~h}-\mathrm{PG} \geq$ $11.1 \mathrm{mmol} / \mathrm{L})$. Vitamin $\mathrm{D}$ status was defined as "deficiency" [25(OH)D $<20 \mathrm{ng} / \mathrm{ml}]$, "insufficiency" [20 ng/ml $\leq 25(\mathrm{OH}) \mathrm{D}<30 \mathrm{ng} / \mathrm{ml}$ and "sufficiency" [25(OH)D $\geq 30 \mathrm{ng} / \mathrm{ml}$ ] [15]. Hypertension was defined as a sitting blood pressure of at least $140 \mathrm{mmHg}$ systolic or $90 \mathrm{mmHg}$ diastolic or anti-hypertensive drug use. Smoking is defined as current smoking of cigarettes or tobacco leaves with 1 branch or $1 \mathrm{~g}$ of tobacco leaves per day; alcohol consumption was defined as drinking at least once a week for more than 1 year. ASCVD events as defined by the WHO diagnostic criteria for MONICA[16] including coronary heart disease events (stable angina pectoris, unstable angina pectoris and acute myocardial infarction and sudden cardiac death, chronic coronary heart disease death) and fatal and nonfatal ischemic cerebral apoplexy, the related diseases were diagnosed by questionnaire survey, telephone follow-up, case information, medical history, medication history and taking photos of medical records.

\section{Statistical analysis}

All analyses were performed using SPSS software version 23.0. The participants were divided into four groups according to quartiles of serum 25(OH)D concentration, instead of the diagnostic criteria of vitamin $D$ deficiency and insufficiency because vitamin $D$ deficiency or insufficiency universally exists in the general population and only $2.0 \%$ had serum $25(\mathrm{OH}) \mathrm{D} \geq 30 \mathrm{ng} / \mathrm{mL}$. Normally distributed continuous variables were expressed as the mean \pm standard deviation (SD), and non-normally distributed variables were presented as medians (interquartile ranges). One-way ANOVA with LSD analysis was used to evaluate differences in quantitative data or Chi-square test with Bonferroni correction for categorical variables. Kruskal-Wallis $H$ test to perform multiple comparisons of non-normally distributed data. Partial correlation analysis were used to explore the correlation between serum 25(OH)D concentration and glucolipid metabolism index. The cumulative incidence of ASCVD in different 25(OH)D level groups and 
in different glucose metabolism groups were calculated and tested by Log-Rank. The linear-by-linear association test and Chi-square test value for trends was used to explore the trend of ASCVD incidence with 25(OH)D level in different glucose metabolism groups. The hazard ratios (HR) and 95\% confidence intervals $(\mathrm{Cl})$ were calculated by multivariate Cox regression analyses. A $p$-value $<0.05$ was considered statistically significant.

\section{Results}

\section{Baseline characteristics of the study participants stratified by 25(OH)D quartiles}

A total of 7061 subjects were included in the study, comprising 2012 males(28.5\%) and 5049 females (71.5\%).The mean age of the study population was 57.6 years ( $57.6 \pm 8.4$ years) and the median (interquartile range) serum 25(OH)D levels was $15.91(11.56,19.98) \mathrm{ng} / \mathrm{mL}$. The prevalence of vitamin $\mathrm{D}$ deficiency $[25(\mathrm{OH}) \mathrm{D}<20 \mathrm{ng} / \mathrm{mL}]$ is $75.1 \%$, merely $2.0 \%$ of those with vitamin $\mathrm{D}$ sufficiency[25(OH)D $\geq 30$ $\mathrm{ng} / \mathrm{mL}$ ]. Table 1 shows the baseline clinical and biochemical parameters according to the quartiles groups for the 25(OH)D. Overall, participants in lower quartile of 25(OH)D were more likely to be older, female, to had a lower smoking and drinking rate and had a higher prevalence of hypertension. Besides, the BMI, FPG, and 2h-PG levels of the study population decreased with the increase of 25(OH)D level (all $P<0.05)$. However, no statistically significant differences were detected between in 25(OH)D quartiles group for levels of HbA1c, HDL-C, LDL-C, TC and TG ( $P>0.05)$.From first quartile to fourth quartile, the prevalence of IGR showed a significant downward trend $(P<0.05)$, and the prevalence of diabetes also showed a weak downward trend, but there was no statistical difference $(P>0.05)$.

Table 1 Baseline characteristics of the study participants stratified by 25(OH)D quartiles 


\begin{tabular}{|c|c|c|c|c|c|}
\hline Characteristics & Total & Quartile 1 & Quartile 2 & Quartile 3 & Quartile 4 \\
\hline cut points(ng/mL) & $1.35-65.69$ & $\leq 11.56$ & $11.57-15.91$ & $15.92-19.98$ & $\geq 19.99$ \\
\hline no. of participants & 7061 & 1767 & 1764 & 1765 & 1765 \\
\hline male vs. female & $2012 / 5049$ & $307 / 1460$ & $447 / 1317^{\mathrm{a}}$ & $528 / 1237^{\mathrm{ab}}$ & $730 / 1035^{\mathrm{abc}}$ \\
\hline age (years) & $57.6 \pm 8.4$ & $58.9 \pm 8.8$ & $57.5 \pm 8.6^{\mathrm{a}}$ & $57.2 \pm 8.0^{\mathrm{a}}$ & $56.9 \pm 8.1^{\mathrm{a}}$ \\
\hline hypertension(\%) & $2525(35.8)$ & $702(39.7)$ & $614(34.8)^{\mathrm{a}}$ & $608(34.4)^{\mathrm{a}}$ & $601(34.1)^{\mathrm{a}}$ \\
\hline smoking $(\%)$ & $1151(16.3)$ & $218(12.3)$ & $276(15.6)^{\mathrm{a}}$ & $285(16.1)^{\mathrm{a}}$ & $372(21.1)^{\mathrm{abc}}$ \\
\hline drinking $(\%)$ & $2008(28.4)$ & $389(22.0)$ & $484(27.4)^{\mathrm{a}}$ & $532(30.1)^{\mathrm{a}}$ & $603(34.2)^{\mathrm{ab}}$ \\
\hline \multicolumn{6}{|c|}{ glucose metabolism status } \\
\hline NGT(\%) & $3272(46.3)$ & $757(42.8)$ & $818(46.4)$ & $827(46.9)$ & $870(49.3)^{\mathrm{a}}$ \\
\hline $\operatorname{IGR}(\%)$ & $2001(28.3)$ & $558(31.6)$ & $499(28.3)$ & $491(27.8)$ & $453(25.7)^{\mathrm{a}}$ \\
\hline $\mathrm{T} 2 \mathrm{DM}(\%)$ & $1788(25.3)$ & $452(25.6)$ & $447(25.3)$ & $447(25.3)$ & $442(25.0)$ \\
\hline $\operatorname{BMI}\left(\mathrm{kg} / \mathrm{m}^{2}\right)$ & $24.17 \pm 3.30$ & $24.38 \pm 3.60$ & $24.21 \pm 3.42$ & $24.06 \pm 3.10^{\mathrm{a}}$ & $24.01 \pm 3.04^{\mathrm{a}}$ \\
\hline $\mathrm{FPG}(\mathrm{mmol} / \mathrm{L})$ & $6.00 \pm 1.71$ & $6.09 \pm 1.84$ & $6.03 \pm 1.76$ & $5.95 \pm 1.57^{\mathrm{a}}$ & $5.94 \pm 1.65^{\mathrm{a}}$ \\
\hline 2h-PG(mmol/L)* & $7.60(6.10,10.00)$ & $7.90(6.28,10.50)$ & $7.60(6.13,9.90)$ & $7.50(6.04,9.86)^{\mathrm{a}}$ & $7.41(6.00,9.70)^{\mathrm{a}}$ \\
\hline $\operatorname{HbA1c}(\%)$ & $6.16 \pm 1.03$ & $6.18 \pm 1.08$ & $6.18 \pm 1.06$ & $6.16 \pm 1.02$ & $6.10 \pm 0.97$ \\
\hline $\mathrm{HDL}-\mathrm{C}(\mathrm{mmol} / \mathrm{L})$ & $1.23 \pm 0.30$ & $1.21 \pm 0.31$ & $1.23 \pm 0.31$ & $1.23 \pm 0.29$ & $1.23 \pm 0.30$ \\
\hline LDL-C $(\mathrm{mmol} / \mathrm{L})$ & $2.56 \pm 0.77$ & $2.55 \pm 0.82$ & $2.56 \pm 0.76$ & $2.59 \pm 0.77$ & $2.56 \pm 0.75$ \\
\hline $\mathrm{TC}(\mathrm{mmol} / \mathrm{L})$ & $4.57 \pm 1.05$ & $4.57 \pm 1.12$ & $4.58 \pm 1.07$ & $4.58 \pm 1.04$ & $4.53 \pm 0.97$ \\
\hline $\mathrm{TG}(\mathrm{mmol} / \mathrm{L})^{*}$ & $1.50(1.07,2.14)$ & $1.53(1.09,2.19)$ & $1.51(1.06,2.15)$ & $1.52(1.05,2.14)$ & $1.45(1.06,2.07)$ \\
\hline $25(\mathrm{OH}) \mathrm{D}(\mathrm{ng} / \mathrm{ml}) *$ & $15.91(11.56,19.98)$ & $9.86(8.66,10.64)$ & $13.81(12.76,14.85)^{\mathrm{a}}$ & $17.89(16.84,18.93)^{a b}$ & $22.70(21.19,24.86)^{\mathrm{abc}}$ \\
\hline
\end{tabular}

*:non-normally distributed variables were presented as medians (interquartile

ranges); ${ }^{\mathrm{a}} P<0.05$ Quartile 2, Quartile 3 or Quartile 4 versus Quartile $1{ }^{\mathrm{b}}{ }^{\mathrm{b}} P<0.05$ Quartile 3 or Quartile 4 versus Quartile 2; ${ }^{\mathrm{c}} P<0.05$ Quartile 4 versus Quartile 3

NGT, normal glucose tolerance; IGR, impaired glucose regulation; T2DM, type 2 diabetes mellitus; BMI, body mass index; FPG, fasting plasma glucose; $2 \mathrm{~h}-\mathrm{PG}, 2 \mathrm{~h}$ postprandial plasma glucose; HbA1c, glycated haemoglobin; HDL-C, high density lipoprotein cholesterol; LDL-C, low-density lipoprotein cholesterol; TC, total cholesterol; TG, triglyceride; 25(OH)D, 25-hydroxyvitamin D

\section{Correlation between serum 25(OH)D concentration and glucolipid metabolism index}

The associations between 25(OH)D concentration and glucolipid metabolism index were examined by the study. The partial correlation analysis showed that serum 25(OH)D concentration was negatively correlated with the levels of FPG, 2h-PG, HbA1c and TG when controlling gender, age, smoking, drinking, $\mathrm{BMI}$, hypertension and diabetes, the partial correlation coefficients were $-0.047,-0.056,-0.035,-0.042$, respectively (all $P<0.05$ ). Meanwhile, serum $25(\mathrm{OH}) \mathrm{D}$ was positively correlated with $\mathrm{HDL}-\mathrm{C}$ and $\mathrm{LDL}-\mathrm{C}$ $(P<0.05)$, but we did not find a correlation between serum levels of $25(\mathrm{OH}) \mathrm{D}$ and TC(Table 2$)$. 
Table 2 Correlation between serum 25(OH)D concentration and glucolipid metabolism index

\begin{tabular}{|c|c|c|c|c|c|c|}
\hline \multirow[t]{2}{*}{ Index } & \multicolumn{2}{|l|}{ Unadjusted } & \multicolumn{2}{|l|}{ Model 1} & \multicolumn{2}{|l|}{ Model 2} \\
\hline & $\begin{array}{l}\text { Partial correlation } \\
\text { coefficient }\end{array}$ & $P$ value & $\begin{array}{l}\text { Partial correlation } \\
\text { coefficient }\end{array}$ & $P$ value & $\begin{array}{l}\text { Partial correlation } \\
\text { coefficient }\end{array}$ & $P$ value \\
\hline$\overline{\mathrm{FPG}(\mathrm{mmol} / \mathrm{L})}$ & -0.035 & 0.003 & -0.048 & 0.000 & -0.047 & 0.000 \\
\hline 2h-PG (mmol/L) & -0.056 & 0.000 & -0.053 & 0.000 & -0.056 & 0.000 \\
\hline $\mathrm{HbA} 1 \mathrm{c}(\%)$ & -0.038 & 0.001 & -0.040 & 0.001 & -0.035 & 0.003 \\
\hline $\mathrm{HDL}-\mathrm{C}(\mathrm{mmol} / \mathrm{L})$ & 0.013 & 0.274 & 0.061 & 0.000 & 0.050 & 0.000 \\
\hline LDL-C(mmol/L) & 0.004 & 0.743 & 0.031 & 0.009 & 0.035 & 0.003 \\
\hline $\mathrm{TC}(\mathrm{mmol} / \mathrm{L})$ & -0.022 & 0.069 & 0.018 & 0.126 & 0.023 & 0.058 \\
\hline TG(mmol/L) & -0.047 & 0.000 & -0.055 & 0.000 & -0.042 & 0.000 \\
\hline
\end{tabular}

Model 1: adjusted for age, gender.

Model 2: adjusted for age, gender, smoking, drinking, body mass index, and history of hypertension and diabetes

FPG, fasting plasma glucose; 2h-PG, $2 \mathrm{~h}$ postprandial plasma glucose; HbA1c, glycated haemoglobin; HDL-C, high density lipoprotein cholesterol; LDL-C, low-density lipoprotein cholesterol; TC, total cholesterol; TG, triglyceride; 25(OH)D, 25-hydroxyvitamin D

\section{Incidence of ASCVD among groups with 25(OH)D quartiles}

After a median follow-up of 3.3 years, a total of 216 patients (3.1\%) experienced ASCVD events, and the ASCVD cumulative incidence in quartiles of $25(\mathrm{OH}) \mathrm{D}$ groups were $4.1 \%, 3.0 \%, 3.1 \%$, and $2.0 \%$, respectively. There was a linear trend toward decreasing ASCVD events as serum 25(OH)D increased. This trend was statistically significant using Log-Rank test $\left(c^{2}=11.676, P=0.009\right)$ Figure 2.

\section{Incidence of ASCVD among groups with 25(OH)D quartiles}

After a median follow-up of 3.3 years, a total of 216 patients (3.1\%) experienced ASCVD events, and the ASCVD cumulative incidence in quartiles of $25(\mathrm{OH}) \mathrm{D}$ groups were $4.1 \%, 3.0 \%, 3.1 \%$, and $2.0 \%$, respectively. There was a linear trend toward decreasing ASCVD events as serum 25(OH)D increased. This trend was statistically significant using Log-Rank test( $\left.c^{2}=11.676, P=0.009\right)$ Figure 2.

Table 3 Hazard ratios for ASCVD events in participants stratified by 25(OH)D quartiles 


\begin{tabular}{lllll}
\hline Model & \multicolumn{1}{c}{ Quartile 1 } & \multicolumn{1}{c}{ Quartile 2 } & \multicolumn{1}{c}{ Quartile 3 } & Quartile 4 \\
\hline Unadjusted & $1.972 \llbracket 1.322-2.942 \square$ & $1.430 \square 0.937-2.184 \square$ & $1.522 \square 1.000-2.317 \square$ & Ref. \\
& $P=0.001$ & $P=0.098$ & $P=0.050$ & \\
Model 1 & $1.902 \square 1.258-2.877 \square$ & $1.458 \square 0.951-2.235 \square$ & $1.561 \square 1.024-2.381 \square$ & Ref. \\
& $P=0.002$ & $P=0.084$ & $P=0.039$ & \\
\hline Model 2 & $1.765 \square 1.165-2.675 \square$ & $1.391 \square 0.907-2.133 \square$ & $1.510 \square 0.989-2.304 \square$ & Ref. \\
& $P=0.007$ & $P=0.130$ & $P=0.056$ & \\
\hline Model 3 & $1.748 \square 1.150-2.656 \square$ & $1.378 \square 0.897-2.118 \square$ & $1.512 \square 0.989-2.312 \square$ & Ref. \\
& $P=0.009$ & $P=0.143$ & $P=0.056$ & \\
\hline
\end{tabular}

Model 1:adjusted for age and gender

Model 2:adjusted for age, gender, smoking, drinking, diabetes, hypertension and body mass index

Model 3: additionally adjusted for fasting plasma glucose, $2 \mathrm{~h}$ postprandial plasma glucose, glycated haemoglobin, high density lipoprotein cholesterol, low-density lipoprotein cholesterol, total cholesterol, triglyceride

\section{Hazard ratios for ASCVD in participants with $10 \mathrm{ng} / \mathrm{ml}$ per increase of 25(OH)D}

Using serum 25(OH)D as a continuous variable $(10 \mathrm{ng} / \mathrm{ml}$ per increase),Cox regression analyses showed that inverse association of the 25(OH)D concentration with the risk of ASCVD(HR:0.696, 95\%Cl:0.545$0.890, P=0.004)$.This association remained statistically significant after adjusted for confounders including age, gender, smoking, drinking, BMl, diabetes, hypertension, FPG, 2hPG, HbA1c, HDL-C, LDL-C, TC and TG (HR:0.761,95\% Cl:0.590-0.980,P = 0.034)(Table 4).

Table 4 Hazard ratios for ASCVD in participants with $10 \mathrm{ng} / \mathrm{ml}$ per increase of 25(OH)D

\begin{tabular}{llllll}
\hline Model & $\beta$ & SE & Wald & $P$ value & HR (95\% CI) \\
\hline Unadjusted & -0.362 & 0.125 & 8.389 & 0.004 & $0.696(0.545-0.890)$ \\
Model 1 & -0.341 & 0.128 & 7.134 & 0.008 & $0.711(0.554-0.913)$ \\
Model 2 & -0.284 & 0.129 & 4.866 & 0.027 & $0.753(0.585-0.969)$ \\
Model 3 & -0.274 & 0.129 & 4.481 & 0.034 & $0.761(0.590-0.980)$
\end{tabular}

Model 1:adjusted for age and gender

Model 2:adjusted for age, gender, smoking, drinking, diabetes, hypertension, and body mass index

Model 3: additionally adjusted for fasting plasma glucose, $2 \mathrm{~h}$ postprandial plasma glucose, glycated haemoglobin, high density lipoprotein cholesterol, low-density lipoprotein cholesterol, total cholesterol, triglyceride

\section{Incidence of ASCVD among populations of different glucose metabolism status within 25(OH)D quartiles}

According to different glucose metabolism status, 7061 individuals were classified into NGT group ( $\mathrm{n}=$ 3272), IGR group ( $n=2001)$ and T2DM group $(n=1788)$. A median follow-up of 3.3 years, the cumulative incidence of ASCVD in the three groups was $1.7 \%, 2.7 \%$, and $5.9 \%$, respectively. There was 
no statistically difference in ASCVD incidence among 25(OH)D quartiles in NGT and IGR groups. Interestingly, among the T2DM population, the incidence rate of ASCVD were 8.8\%, 5.1\%, 6.0\%, and $3.6 \%$, respectively, from the first quartile of serum $25(\mathrm{OH}) \mathrm{D}$ to fourth. Overall $邓$ the incidence of ASCVD showed a downward trend with the increase of $25(\mathrm{OH}) \mathrm{D}$ level in patients with $\mathrm{T} 2 \mathrm{DM}(P=0.003)$ Figure 3.

\section{Hazard ratios for ASCVD events in T2DM patients according to 25(OH)D quartiles}

Compared with the fourth quartile in T2DM patients, those in first quartile had a higher risk of ASCVD incidence $(P=0.002)$. After adjusted for all confounding factors, the trend was still significant, and the hazard ratio of ASCVD events $(95 \% \mathrm{CI})$ was 2.284(1.242-4.202)in the lowest quartile than those in the highest $(P=0.008)$ Table 5 .

Table 5 Hazard ratios for ASCVD in T2DM patients according to 25(OH)D quartiles

\begin{tabular}{lllll}
\hline Model & \multicolumn{1}{c}{ Quartile 1 } & \multicolumn{1}{c}{ Quartile 2 } & \multicolumn{1}{c}{ Quartile 3 } & Quartile 4 \\
\hline Unadjusted & $2.481 \square 1.389-4.430 \square$ & $1.373 \llbracket 0.725-2.599 \square$ & $1.666 \square 0.897-3.091 \square$ & Ref. \\
& $P=0.002$ & $P=0.330$ & $P=0.106$ & \\
Model 1 & $2.614 \llbracket 1.434-4.765 \square$ & $1.455 \square 0.765-2.7668 \square$ & $1.759 \square 0.945-3.275 \square$ & Ref. \\
& $P=0.002$ & $P=0.253$ & $P=0.075$ & \\
\hline Model 2 & $2.317 \square 1.269-4.232 \square$ & $1.370 \square 0.720-2.607 \square$ & $1.738 \square 0.933-3.239 \square$ & Ref. \\
& $P=0.006$ & $P=0.338$ & $P=0.082$ & \\
\hline Model 3 & $2.284 \square 1.242-4.202 \square$ & $1.338 \square 0.699-2.560 \square$ & $1.736 \square 0.928-3.247 \square$ & Ref. \\
& $P=0.008$ & $P=0.379$ & $P=0.084$ & \\
\hline
\end{tabular}

Model 1:adjusted for age and gender

Model 2:adjusted for age, gender, smoking, drinking, hypertension, and body mass index

Model 3: additionally adjusted for fasting plasma glucose, $2 \mathrm{~h}$ postprandial plasma glucose, glycated haemoglobin, high density lipoprotein cholesterol, low-density lipoprotein cholesterol, total cholesterol, triglyceride

\section{Hazard ratios for ASCVD in T2DM patients with per $10 \mathrm{ng} / \mathrm{ml}$ increase of 25(OH)D}

The results showed that for per $10 \mathrm{ng} / \mathrm{ml}$ increase in basline serum 25(OH)D concentrations, the risk of onset ASCVD decreased by 40 percent approximately in T2DM patients(HR:0.597, 95\% Cl:0.415$0.857, P=0.005$ ) when without adjusted for relevant risk factors. After further adjusted for confounding factors, we found that the risk of ASCVD still decreased gradually. In other words, the risk of ASCVD is reduced by $35 \%$ for each $10 \mathrm{ng} / \mathrm{ml}$ elevation of $25(\mathrm{OH}) \mathrm{D}(\mathrm{HR}: 0.649,95 \% \mathrm{Cl}: 0.444-0.948, P=0.025)$ Table 6 .

Table 6 Hazard ratios for ASCVD in T2DM patients with per $10 \mathrm{ng} / \mathrm{ml}$ increase of 25(OH)D 


\begin{tabular}{llllll}
\hline Model & $\beta$ & SE & Wald & $P$ value & HR (95\% CI) \\
\hline Unadjusted & -0.516 & 0.185 & 7.814 & 0.005 & $0.597(0.415-0.857)$ \\
Model 1 & -0.548 & 0.192 & 8.127 & 0.004 & $0.578(0.397-0.843)$ \\
Model 2 & -0.450 & 0.192 & 5.512 & 0.019 & $0.637(0.438-0.928)$ \\
Model 3 & -0.433 & 0.194 & 4.995 & 0.025 & $0.649(0.444-0.948)$ \\
\hline
\end{tabular}

Model 1:adjusted for age and gender

Model 2:adjusted for age, gender, smoking, drinking, hypertension, and body mass index

Model 3: additionally adjusted for fasting plasma glucose, $2 \mathrm{~h}$ postprandial plasma glucose, glycated haemoglobin, high density lipoprotein cholesterol, low-density lipoprotein cholesterol, total cholesterol, triglyceride

\section{Discussion}

In our 3.3-year longitudinal observational follow-up study, we found that vitamin D deficiency is common among middle-aged and elderly populations in Lanzhou, Gansu province. Low levels of serum vitamin D is independently associated with increased risk of ASCVD and the ASCVD risk significantly reduced as serum 25(OH)D levels increased after adjusted for confounders in the subjects, especially in T2DM patients.

Vitamin D deficiency is widespread worldwide. The Rotterdam Study, a population-based cohort of middle-aged and elderly adults in the Netherlands, analyzed data from 3240 people aged 55 years or older who did not have type 2 diabetes mellitus at baseline,the results showed that $56.6 \%$ of the participants were vitamin D deficient[17]. Lahner et al.[18]analyzed the status of vitamin D levels and the prevalence of vitamin $D$ deficiency in middle-aged and elderly people in metropolitan areas of western Germany. They found that overall median vitamin D level was $19.8 \mathrm{ng} / \mathrm{ml}$ in 4149 participants aged 45 to 75 years, with women having lower levels than men and the prevalence of vitamin D deficiency $(<20$ $\mathrm{ng} / \mathrm{ml}$ ) was $50.6 \%$. Whereas, in our study, the median serum $25(\mathrm{OH}) \mathrm{D}$ level of middle-aged and elderly people in Lanzhou urban district was $15.91 \mathrm{ng} / \mathrm{mL}$, significantly lower than German residents.

Additionally, in the present population, the prevalence of vitamin D deficiency was $75.1 \%$, which was more common in women than men $(79.6 \%$ vs $63.8 \%)$, and merely $2.0 \%$ of those with vitamin D sufficiency. Compared to Lahner et al's study[18], our study showed a higher prevalence of vitamin D deficiency and demonstrated significant gender differences. Such differences may be attributed to differences in geographical, skin color, and nutritional status[3]. Our findings supported by two large-scale investigations in Beijing and Shanghai, show that as high as $70 \%$ to $90 \%$ of the participants had vitamin D deficiency[19,20].Although there are slight differences in vitamin $D$ deficiency in various regions of China[21], the overall indication is that there is a high prevalence of vitamin D deficiency. Therefore, active screening of vitamin D levels and appropriate vitamin D supplementation may improve this situation.

A growing number of studies have reported that vitamin $D$ deficiency is associated with many chronic diseases, including ASCVD[22,23,24].A large meta-analysis of observational studies $(n=849,412)$ found 
that low concentrations of 25(OH)D were associated with an increased risk of CVD, the pooled relative risk was 1.35 folds for death from CVD in lowest 25(OH)D tertile compared with the highest tertile, and each $10 \mathrm{nmol} / \mathrm{L}$ reduction in 25(OH)D was associated with a 16\% increase in all-cause mortality[25]. A dose-response meta-analysis of observational studies also reached similar conclusion, which included 32 publications, observed an inverse association between serum 25(OH)D and total cardiovascular events and mortality, particularly in $25(\mathrm{OH}) \mathrm{D}<25 \mathrm{ng} / \mathrm{mL}$, cardiovascular mortality rate increased by $57 \%$. Moreover, per $10 \mathrm{ng} / \mathrm{ml}$ increase in serum $25(\mathrm{OH}) \mathrm{D}$, there was a decrease of $10 \%$ in total cardiovascular events, $12 \%$ in cardiovascular mortality[11]. Based on the above research background, this prospective study discussed the correlation between the serum 25(OH) D level and the risk of ASCVD in Lanzhou community in northwest China. After 3.3 years of follow-up, the cumulative incidence of ASCVD from lowest of $25(\mathrm{OH}) \mathrm{D}$ levels to the highest were $4.1 \%, 3.0 \%, 3.1 \%$ and $2.0 \%$, respectively, and showing a marked decline trend. After further adjusted confounders, we found that those with 25(OH)D lowest $(<11.56 \mathrm{ng} / \mathrm{mL}$ ) had 1.75 folds the risk of ASCVD than those highest $(>19.99 \mathrm{ng} / \mathrm{mL})$ in participants. Additionally, per $10 \mathrm{ng} / \mathrm{ml}$ increase in serum 25(OH)D at baseline, ASCVD risk was reduced by $24 \%$, suggested that extremely low levels of $25(\mathrm{OH}) \mathrm{D}$ is significantly associated with increased incidence of ASCVD events in population of Lanzhou. Our study's results are similar to those mentioned above and also supported by Chen et al[26] . However, compared with previous studies, our study further analyzed the incidence of ASCVD under different glucose metabolism status and its relationship with baseline vitamin D levels. Interestingly, we found that the 3.3 years cumulative incidence of ASCVD in T2DM patients was significantly higher than subjects with NGT and IGR (5.9\% vs $1.7 \%, 5.9 \%$ vs $2.7 \%)$. As we all know, the risk of ASCVD in T2DM patients is 2-4 times than that of non-diabetic patients[27], our study further confirmed the close correlation between ASCVD and T2DM. Moreover, after further comparison of ASCVD risk among people with different glucose metabolism status at 25(OH)D quartiles, we didn't observed that vitamin D levels related to ASCVD incidence in IGR and NGT populations. In contrast, those with 25(OH) D lowest had 2.3 folds risk of ASCVD than those highest in the T2DM population. This result were supported by a prospective cohort study from abroad[28], which evaluated the predictive value of serum $25(\mathrm{OH}) \mathrm{D}$ for improving coronary heart disease $(\mathrm{CHD})$ risk stratification in patients with T2DM. A total of 2,607 patients with T2DM were followed for a median of 8.5 years, and the results showed that the incidence rate of $\mathrm{CHD}$ decreased as serum 25(OH)D increased and patients in the lowest quartile experienced more CHD events. Additionally, per $10 \mathrm{ng} / \mathrm{ml}$ increase in serum 25(OH)D at baseline, ASCVD risk in T2DM patients was reduced by 35\%. The follow-up time of our study was shorter than that of the above research, but the results were consistent basically, besides, our study further using serum 25(OH)D as a continuous variable and analyzed the correlation between 25(OH)D and ASCVD risk in patients of T2DM. The results demonstrated that for each $10 \mathrm{ng} / \mathrm{ml}$ increase of 25(OH)D, ASCVD risk was reduced by $35 \%$, suggested that extremely low level of $25(\mathrm{OH}) \mathrm{D}$ is significantly associated with increased incidence of ASCVD events in T2DM population in Lanzhou.

Although the mechanisms underlying this association are not fully elucidated, it is thought that vitamin $D$ deficiency primarily affects ASCVD risk factors such as inflammation, hypertension and diabetes[29,30]. When vitamin D in the deficient state, the expressions of inflammatory factors INF- $y$, IL-2, IL-6 and TNF-

Page 12/20 
a increased, and the flow rate of mononuclear cells in peripheral blood slowed down, which made the white blood cells more likely to aggregate and adhere to the endothelial cells, and promoted the formation of atherosclastic plaque[31]. In addition, vitamin D can negatively regulate the renin-angiotensin-aldosterone system(RAAS) and play a vital role in maintain blood pressure and protect cardiovascular system in physiological condition[22]. While decreased serum 25(OH)D weakens the inhibitory effect on the RAAS system, and the synthetic secretion of renin and Ang囚increases. When combined with its receptor, Ang囚can activate the expressions of oxidative stress-related proteins including nitrous oxides(NOX)-2, NOX-4, and elevate levels of reactive oxygen species (ROS), damage vascular endothelial cells and cause blood pressure elevated ultimately [32].

Hypertension, hyperglycemia and dyslipidemia are known to be major risk factors for developing $\operatorname{ASCVD}[33,34]$. Recently, considerable evidence suggests that vitamin D has beneficial effects on the cardiovascular system in part by improving the blood lipid profiles, controlling glucose levels and decreasing blood pressure[35,36,37]. Studies have found that vitamin D levels are negatively correlated with blood pressure and is an independent risk factors for hypertension, in addtion, there are differences in vitamin D levels between hypertensive patients and non-hypertensives, and the prevalence of hypertension is increased in people with low vitamin $D$ level[38]. We also found that with the increase of 25(OH)D levels, the prevalence of hypertension showed a downward trend, but the specific causal relationship between blood pressure and vitamin D levels was not further analyzed in this study. A study by Yin et al.[39] on vitamin $D$ and metabolic syndrome in Chinese elderly healthy population showed that vitamin D was inversely related to FPG levels. Cross-sectional study have found that individuals with low serum 25(OH)D have higher LDL-C, TG and TC, and lower HDL-C compared to those with higher levels of $25(\mathrm{OH}) \mathrm{D}[40]$. There are slightly different from previous studies, our study showed that serum $25(\mathrm{OH}) \mathrm{D}$ concentrations were inversely associated with glucolipid metabolism parameters including TG, FPG, PPG and $\mathrm{HbA} 1 \mathrm{C}$ and positively correlation with $\mathrm{HDL}-\mathrm{C}$ and $\mathrm{LDL}-\mathrm{C}$ after control confounders. A meta-analysis backs up our findings, which investigated the effect of vitamin $D$ supplementation and increased serum 25(OH)D concentrations on CVD risk factors, demonstrating that vitamin D supplements resulted in significant reductions in systolic blood pressure, diastolic blood pressure, total TG, TC,LDL and a significant increase in $\mathrm{HDL}$, indicated that maintain vitamin $\mathrm{D}$ sufficiency can decrease the risk factors of ASCVD[41].

This study is a prospective cohort study to explore the association between 25(OH)D levels and the risks of ASCVD incidence in the Chinese population with a 3.3 years follow-up duration. The advantage of our study lie in our adopted strict investigation methods and quality control measures and have a large sample size. Furthermore, the ASCVD outcome was determined using rigorous and detailed clinical investigation. However,we also recognize several limitations of this study: firstly, the short follow-up time may lead to the failure of vitamin D's influence on the occurrence and development of ASCVD; secondly, we did not conduct an intervention study to further clarify the impact of vitamin D supplementation on ASCVD risk; in addition, the correlation between VDR gene polymorphism and the incidence of ASCVD in local population is not confirmed in present study. 


\section{Conclusions}

To sum up, these real-world data showed a high prevalence of vitamin D deficiency, as well as high incidence of ASCVD in middle-aged and elderly people in Lanzhou, China, especially in T2DM patients. This study further found that extremely low serum 25(OH)D levels are independently associated with an increased risk of ASCVD, the increase in $25(\mathrm{OH})$ D levels could reduce the risk of ASCVD, particularly in patients of T2DM.

\section{Abbreviations}

ASCVD:atherosclerotic cardiovascular disease; 25(OH)D:25-hydroxyvitamin D; T2DM: type 2 diabetes mellitus; NGT:normal glucose tolerance; IGR:impaired glucose regulation; CVD:cardiovascular diseases; CHD:coronary heart disease; BMl:body mass index; FPG:fasting plasma glucose; $2 \mathrm{~h}-\mathrm{PG}$ :2h plasma glucose; HbA1c:glycated haemoglobin; TC:total cholesterol; TG:triglycerides; HDL-C:high-density lipoprotein cholesterol; LDL-C:Iow-density lipoprotein cholesterol; RAAS:renin-angiotensinaldosterone system; NOX: nitrous oxides; ROS:reactive oxygen species

\section{Declarations}

\section{Ethics approval and consent to participate}

Our study approved by the ethics committee of Shanghai Jiao Tong University. All study participants provided written informed consent.

\section{Consent for publication}

Not applicable.

\section{Availability of data and materials}

The datasets used and/or analyzed during the current study are not publicly available but are available from the corresponding author on reasonable request.

\section{Competing interests}

The authors declare that they have no competing interests.

\section{Funding}

The present work was supported by the Special Funds of Science and Technology Development of the Chinese Central Government to guide Local in 2020(20JR10FA667), the Diabetes Clinical Research Project of Shanghai Medical and Health Development Foundation (Phase I 10 Study) (DMRFP-I-10)and the Special Research Fund of Standardized Metabolic Disease Management Center (2018-mmczxjj-3). 


\section{Authors' contributions}

SS and D-HZ completed the project, analyzed the data, and drafted the manuscript. X-LT, C-HG designed the study, interpreted the data and contributed to critically revising the manuscript. DZ and Q-MW interpreted the data and contributed to revising the manuscript. NZ, H-LL, L-TW,H-XC, J-JL, L-JL and S-BF contributed to recruitment of subjects and follow-up surveys $₫$ All authors read and approved the final manuscript.

\section{Acknowledgements}

The authors thank all the staf and participants of this study for their important contributions.

\section{Authors' information}

${ }^{1}$ First School of Clinical Medicine, Lanzhou University, 1 Donggang West Road, Lanzhou 730000, Gansu, Peoples' Republic of China; ${ }^{2}$ Department of Endocrinology, the First Hospital of Lanzhou University, 1 Donggang West Road, Lanzhou 730000, Gansu, Peoples' Republic of China; ${ }^{3}$ Department of Endocrinology, the Third People's Hospital, 763 Duanjiatan Road, Lanzhou 730000, Gansu, Peoples' Republic of China.

\section{References}

1. Roth GA, Johnson C, Abajobir A, Abd-Allah F, Abera SF, Abyu G, Ahmed M, Aksut B, Alam T, Alam K, et al. Global, Regional, and National Burden of Cardiovascular Diseases for 10 Causes, 1990 to 2015. J Am Coll Cardiol. 2017;70(1):1-25. doi: 10.1016/j.jacc.2017.04.052.

2. Weng W, Tian Y, Kong SX, Ganguly R, Hersloev M, Brett J, Hobbs T. The prevalence of cardiovascular disease and antidiabetes treatment characteristics among a large type 2 diabetes population in the United States. Endocrinol Diabetes Metab. 2019;2(3):e00076. doi: 10.1002/edm2.76.

3. Chamberlain JJ, Johnson EL, Leal S, Rhinehart AS, Shubrook JH, Peterson L. Cardiovascular Disease and Risk Management: Review of the American Diabetes Association Standards of Medical Care in Diabetes 2018. Ann Intern Med. 2018;168(9):640-650. doi: 10.7326/M18- 0222.

4. Bikle DD. Vitamin D metabolism, mechanism of action, and clinical applications. Chem Biol,2014,21(3):319-329.

5. Cashman Kevin D,Vitamin D Deficiency: Defining, Prevalence, Causes, and Strategies of Addressing.Calcif Tissue Int, 2020, 106(1): 14-29.

6. Holick MF. The vitamin D deficiency pandemic: Approaches for diagnosis, treatment and prevention. Rev Endocr Metab Disord, 2017, 18(2):153-165.

7. Stoffels K, Overbergh L, Giulietti A, Verlinden L, Bouillon R, Mathieu C. Immune regulation of 25hydroxyvitamin-D3-1alpha-hydroxylase in human monocytes. J Bone Miner Res. 2006;21(1):37-47. 
doi: $10.1359 / J B M R .050908$.

8. Chen S, Law CS, Gardner DG. Vitamin D-dependent suppression of endothelin-induced vascular smooth muscle cell proliferation through inhibition of CDK2 activity. J Steroid Biochem Mol Biol. 2010;118(3):135-41. doi: 10.1016/j.jsbmb.2009.11.002.

9. Duarte C, Carvalheiro H, Rodrigues AM, Dias SS, Marques A, Santiago T, Canhão H, Branco JC, da Silva JAP. Prevalence of vitamin D deficiency and its predictors in the Portuguese population: a nationwide population-based study. Arch Osteoporos. 2020;15(1):36. doi: 10.1007/s11657-020-0695$\mathrm{x}$.

10. Welles CC, Whooley MA, Karumanchi SA, Hod T, Thadhani R, Berg AH, Ix JH, Mukamal KJ. Vitamin D deficiency and cardiovascular events in patients with coronary heart disease: data from the Heart and Soul Study. Am J Epidemiol. 2014;179(11):1279-87. doi: 10.1093/aje/kwu059.

11. Zhang R, Li B, Gao X, Tian R, Pan Y, Jiang Y, Gu H, Wang Y, Wang Y, Liu G. Serum 25-hydroxyvitamin $\mathrm{D}$ and the risk of cardiovascular disease: dose-response meta-analysis of prospective studies. Am $\mathrm{J}$ Clin Nutr. 2017;105(4):810-819. doi: 10.3945/ajen.116.140392.

12. Manson JE, Cook NR, Lee IM, Christen W, Bassuk SS, Mora S, Gibson H, Gordon D, Copeland T, D'Agostino D, et al. Vitamin D Supplements and Prevention of Cancer and Cardiovascular Disease. N Engl J Med. 2019;380(1):33-44. doi: 10.1056/NEJMoa1809944.

13. Bi Y, Lu J, Wang W, Mu Y, Zhao J, Liu C, Chen L, Shi L, Li Q, Wan Q, et al. Cohort profile: risk evaluation of cancers in Chinese diabetic individuals: a longitudinal (REACTION) study. J Diabetes. 2014;6(2):147-57. doi: 10.1111/1753-0407.12108.

14. Puavilai G, Chanprasertyotin S, Sriphrapradaeng A. Diagnostic criteria for diabetes mellitus and other categories of glucose intolerance: 1997 criteria by the Expert Committee on the Diagnosis and Classification of Diabetes Mellitus (ADA), 1998 WHO consultation criteria, and 1985 WHO criteria. World Health Organization. Diabetes Res Clin Pract. 1999;44(1): 21-6. doi: 10.1016/s01688227(99)00008-x.

15. Holick MF, Binkley NC, Bischoff-Ferrari HA, Gordon CM, Hanley DA, Heaney RP, Murad MH, Weaver CM; Endocrine Society. Evaluation, treatment, and prevention of vitamin D deficiency: an Endocrine Society clinical practice guideline. J Clin Endocrinol Metab. 2011;96(7):1911-30. doi: 10.1210/jc.2011-0385.

16. WHO MONICA Project Principal Investigators. The World Health Organization MONICA Project (monitoring trends and determinants in cardiovascular disease): a major international collaboration. WHO MONICA Project Principal Investigators. J Clin Epidemiol, 1988, 41(2): 105-114.

17. Vitezova A, Zillikens MC, van Herpt TT, Sijbrands EJ, Hofman A, Uitterlinden AG, Franco OH, Kiefte-de Jong JC. Vitamin D status and metabolic syndrome in the elderly: the Rotterdam Study. Eur J Endocrinol. 2015;172(3):327-35. doi: 10.1530/EJE-14-0580.

18. Lahner H, Schramm S, Joeckel KH, Erbel R, Mann K, Führer D, Moebus S;Heinz Nixdorf Recall study Investigative Group. Prevalence of vitamin D deficiency in a population-based study: Results from the Heinz Nixdorf Recall Study. Exp Clin Endocrinol Diabetes,2013, 121(3):10-64. 
19. Lu L, Yu Z, Pan A, Hu FB, Franco OH, Li H, Li X, Yang X, Chen Y, Lin X. Plasma 25-hydroxyvitamin D concentration and metabolic syndrome among middle-aged and elderly Chinese individuals. Diabetes Care. 2009;32(7):1278-83. doi: 10.2337/dc09-0209.

20. Zhao J, Xia W, Nie M, Zheng X, Wang Q, Wang X, Wang W, Ning Z, Huang W, Jiang Y, et al. The levels of bone turnover markers in Chinese postmenopausal women: Peking Vertebral Fracture study. Menopause. 2011;18(11):1237-43. doi: 10.1097/gme.0b013e31821d7ff7.

21. Wang T, Sun H, Ge H, Liu X, Yu F, Han H, Wang J, Li W. Association between vitamin D and risk of cardiovascular disease in Chinese rural population. PLoS One. 2019;14(5):e0217311. doi: 10.1371/journal.pone.0217311.

22. Hiemstra T, Lim K, Thadhani R, Manson JE. Vitamin D and Atherosclerotic Cardiovascular Disease. J Clin Endocrinol Metab(2019).doi: 10.1210/jc.2019-00194.

23. van de Luijtgaarden KM, Voûte MT, Hoeks SE, Bakker EJ, Chonchol M, Stolker RJ, Rouwet EV, Verhagen HJ. Vitamin D deficiency may be an independent risk factor for arterial disease. Eur J Vasc Endovasc Surg. 2012;44(3):301-6. doi: 10.1016/j.ejvs.2012.06.017.

24. Aljefree NM, Lee P, Alsaqqaf JM, Ahmed F. Association between Vitamin D Status and Coronary Heart Disease among Adults in Saudi Arabia: A Case-Control Study. Healthcare (Basel). 2016;4(4):77. doi: 10.3390/healthcare4040077.

25. Chowdhury R, Kunutsor S, Vitezova A, Oliver-Williams C, Chowdhury S, Kiefte-de-Jong JC, Khan H, Baena CP, Prabhakaran D, Hoshen MB, et al. Vitamin D and risk of cause specific death: systematic review and meta-analysis of observational cohort and randomised intervention studies. BMJ. 2014;348:g1903. doi: 10.1136/bmj.g1903.

26. Chen FH, Liu T, Xu L, Zhang L, Zhou XB. Association of Serum Vitamin D Level and Carotid Atherosclerosis: A Systematic Review and Meta-analysis. J Ultrasound Med. 2018;37(6):1293-1303. doi: 10.1002/jum.14494.

27. Iglay K, Hannachi H, Joseph Howie P, Xu J, Li X, Engel SS, Moore LM, Rajpathak S. Prevalence and co-prevalence of comorbidities among patients with type 2 diabetes mellitus. Curr Med Res Opin. 2016;32(7):1243-52. doi: 10.1185/03007995.2016.1168291.

28. Heidari B, Nargesi AA, Hafezi-Nejad N, Sheikhbahaei S, Pajouhi A, Nakhjavani M, Esteghamati A. Assessment of serum 25-hydroxy vitamin D improves coronary heart disease risk stratification in patients with type 2 diabetes. Am Heart J. 2015;170(3):573-9.e5. doi: 10.1016/j.ahj.2015.06.017.

29. Liu L, Chen M, Hankins SR, Nùñez AE, Watson RA, Weinstock PJ, Newschaffer CJ, Eisen HJ; Drexel Cardiovascular Health Collaborative Education, Research, and Evaluation Group. Serum 25hydroxyvitamin D concentration and mortality from heart failure and cardiovascular disease, and premature mortality from all-cause in United States adults. Am J Cardiol. 2012;110(6):834-9. doi: 10.1016/j.amjcard.2012.05.013.

30. Isnard RN, Pannier BM, Laurent S, London GM, Diebold B, Safar ME. Pulsatile diameter and elastic modulus of the aortic arch in essential hypertension: a noninvasive study. J Am Coll Cardiol. 1989;13(2):399-405. doi: 10.1016/0735-1097(89)90518-4. 
31. Bozic M, Álvarez Á, de Pablo C, Sanchez-Niño MD, Ortiz A, Dolcet X, Encinas M, Fernandez E, Valdivielso JM. Impaired Vitamin D Signaling in Endothelial Cell Leads to an Enhanced LeukocyteEndothelium Interplay: Implications for Atherosclerosis Development. PLoS One. 2015;10(8):e0136863. doi: 10.1371/journal.pone.0136863.

32. Dong J, Wong SL, Lau CW, Lee HK, Ng CF, Zhang L, Yao X, Chen ZY, Vanhoutte PM, Huang Y. Calcitriol protects renovascular function in hypertension by down-regulating angiotensin II type 1 receptors and reducing oxidative stress. Eur Heart J. 2012;33(23): 2980-90. doi: 10.1093/eurheartj/ehr459.

33. Jellinger PS, Smith DA, Mehta AE, Ganda O, Handelsman Y, Rodbard HW, Shepherd MD, Seibel JA; AACE Task Force for Management of Dyslipidemia and Prevention of Atherosclerosis. American Association of Clinical Endocrinologists' Guidelines for Management of Dyslipidemia and Prevention of Atherosclerosis: executive summary. Endocr Pract. 2012;18(2):269-93. doi: 10.4158/ep.18.2.269.

34. Li T, Zhu J, Fang Q, Duan X, Zhang M, Diao S, Zhou Y, Yang S, Kong Y, Cai X. Association of H-Type Hypertension with Stroke Severity and Prognosis. Biomed Res Int. 2018;2018:8725908. doi: 10.1155/2018/8725908.

35. Wacker M, Holick MF. Vitamin D - effects on skeletal and extraskeletal health and the need for supplementation. Nutrients. 2013;5(1):111-48. doi: 10.3390/nu5010111.

36. Abu el Maaty MA, Gad MZ. Vitamin D deficiency and cardiovascular disease: potential mechanisms and novel perspectives. J Nutr Sci Vitaminol (Tokyo). 2013;59(6):479-88. doi: 10.3177/jnsv.59.479.

37. Vacek JL, Vanga SR, Good M, Lai SM, Lakkireddy D, Howard PA. Vitamin D deficiency and supplementation and relation to cardiovascular health. Am J Cardiol. 2012;109(3): 359-63. doi: 10.1016/j.amjcard.2011.09.020.

38. Scragg R, Sowers M, Bell C. Serum 25-hydroxyvitamin D, ethnicity, and blood pressure in the Third National Health and Nutrition Examination Survey. Am J Hypertens, 2007;20 (7) :713-719.

39. Yin X, Sun Q, Zhang X, Lu Y, Sun C, Cui Y, Wang S. Serum 25(OH)D is inversely associated with metabolic syndrome risk profile among urban middle-aged Chinese population. Nutr J. 2012;11:68. doi: 10.1186/1475-2891-11-68.

40. Skaaby T, Husemoen LL, Pisinger C, Jørgensen T, Thuesen BH, Fenger M, Linneberg A. Vitamin D status and changes in cardiovascular risk factors: a prospective study of a general population. Cardiology. 2012;123(1):62-70. doi: 10.1159/000341277.

41. Mirhosseini N, Rainsbury J, Kimball SM. Vitamin D Supplementation, Serum 25(OH)D Concentrations and Cardiovascular Disease Risk Factors: A Systematic Review and Meta- Front Cardiovasc Med. 2018;5:87.doi:10.3389/fcvm.2018.00087

\section{Figures}




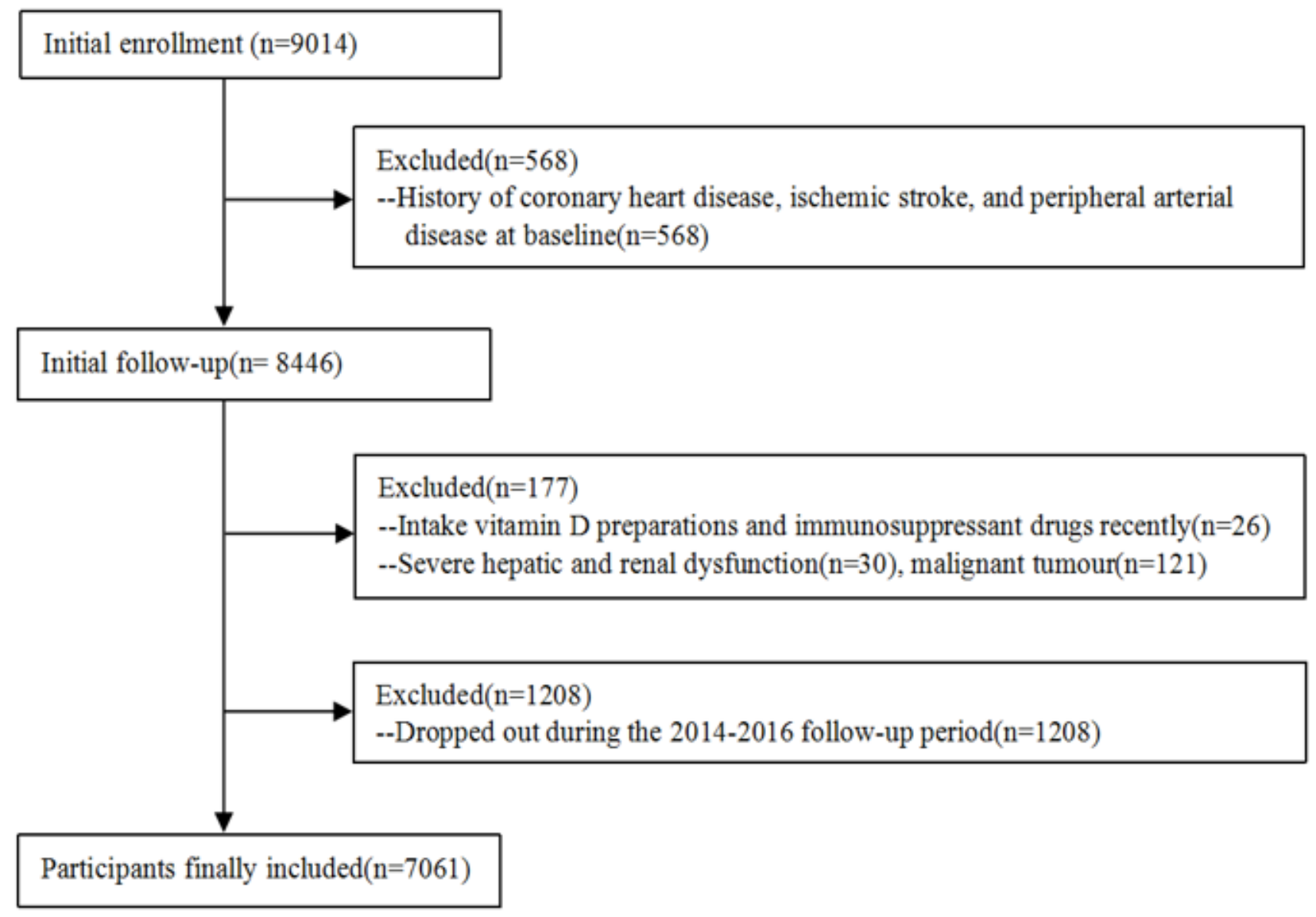

\section{Figure 1}

Flow chart of study population

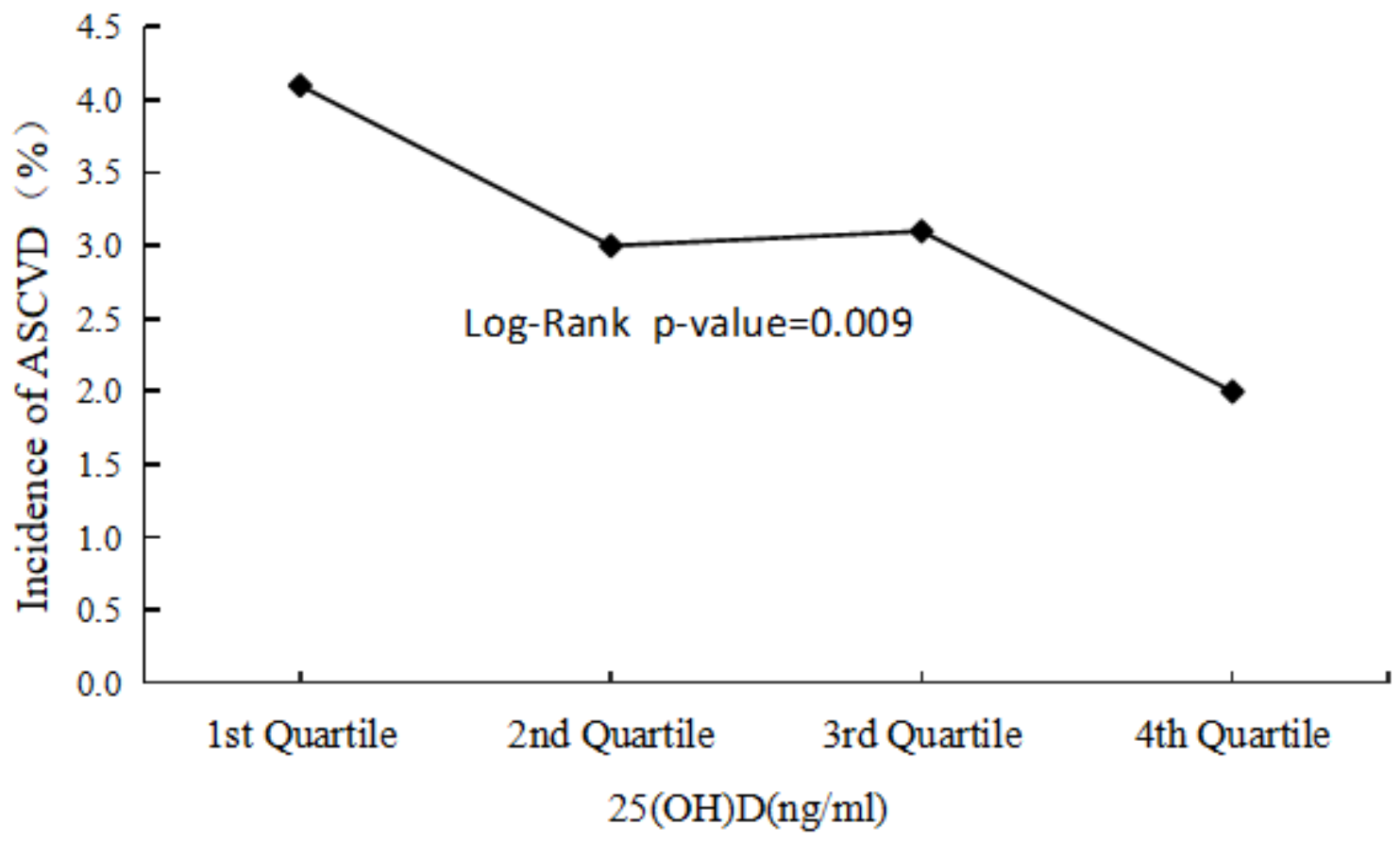


Figure 2

Incidence of ASCVD among groups with 25(OH)D quartiles

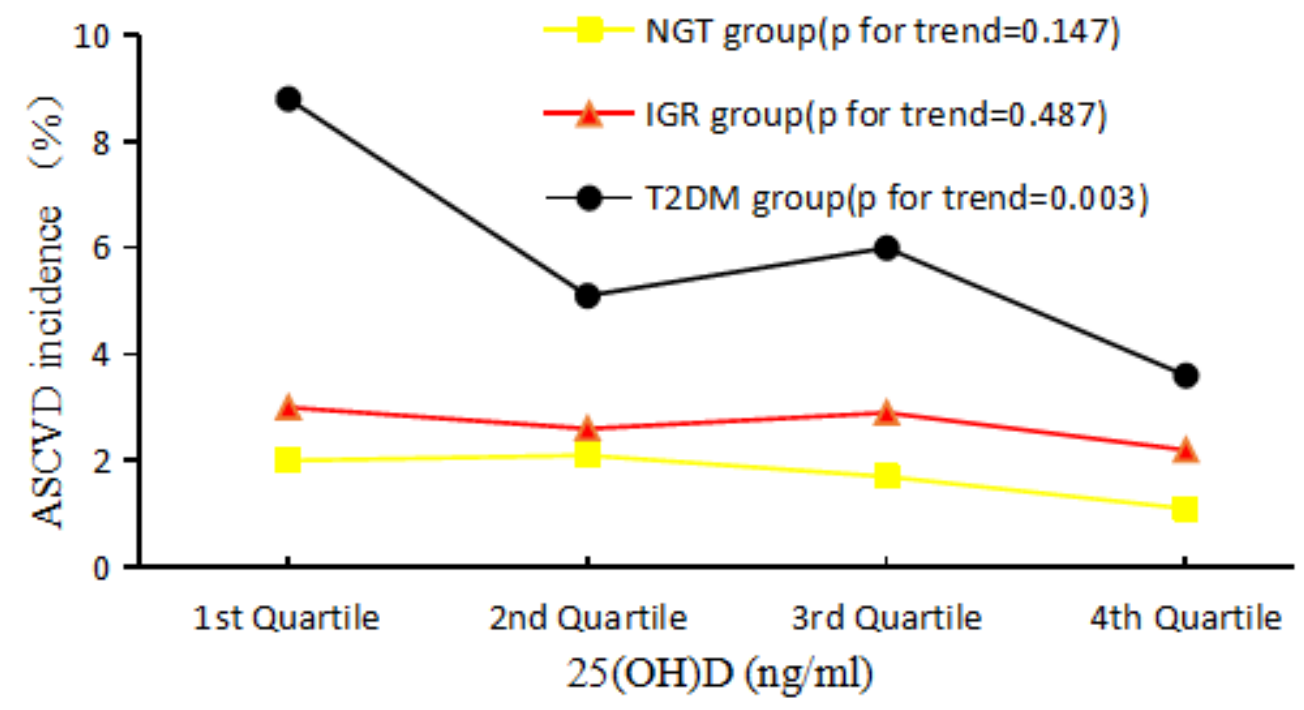

Figure 3

Incidence of ASCVD among populations of different glucose metabolism status within 25(OH)D quartiles 